\title{
Cytokine Gene Polymorphisms and Chromosome 13 Deletion in Multiple Myeloma Patients
}

Multiple Myeloma Hastalarında Sitokin Gen Polimorfizmleri ve Kromozom 13 Delesyonu

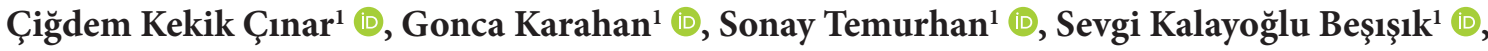
Fatma Savran Oğuz ${ }^{1} \oplus$, Filiz Aydın ${ }^{1}$

1 Istanbul University, Istanbul Faculty of Medicine, Department of Medical Biology, Istanbul, Turkey

ORCID: C..K.Ç. 0000-0003-2098-381X;

G.K. 0000-0001-5339-7126;

S.T. 0000-0001-9889-9330;

S.K.B. 0000-0002-9310-1278;

F.S.O. 0000-0002-6018-8936

F.A. $0000-0001-5984-7538$

Corresponding author/Sorumlu yazar: Çiğdem Kekik Çınar,

Istanbul University, Istanbul Faculty of Medicine, Department of Medical Biology, Istanbul, Turkey

E-mail: citcim@gmail.com

Submitted/Geliș tarihi: 29.09 .2020 Accepted/Kabul Tarihi: 23.10.2020

Citation/Atıf: Kekik Cınar C, Karahan G, Temurhan S, Kalayoglu Besisik S, Savran Oguz F, Aydin F . Cytokine gene polymorphisms and chromosome 13 deletion in Multiple myeloma patients. Sağlık Bilimlerinde İleri Araştırmalar Dergisi 2020; 3(3): 179-185. https://doi.org/10.26650/JARHS2020-653043

\section{ABSTRACT}

Objective: Multiple myeloma (MM) is a clonal enlargement of plasma cells. The most common cytogenetic anomaly in MM is monosomy 13 and appears approximately $40 \%-50 \%$ in multiple myeloma. Studies have shown that in the majority of patients, $80 \%-90 \%$ of chromosomal anomalies are monosomy 13 , and $10 \%-20 \%$ are regional deletions. Monosomy 13 is the most powerful predictor of survival in MM. Cytokines are proteins that regulate many functions of these cells and are secreted by cells of the immune system. Plasma cell stimulates angiogenesis by increasing the release of cytokines such as IL-1 $\beta$, IL-6, and IL-10. Single nucleotide polymorphisms (SNP) of cytokine genes can affect their secretion rate or biological activity. Two of the important factors for MM disease are cytokines and chromosome 13 deletion (del13). We investigate chromosome 13 deletion and cytokine gene polymorphisms (IFN- $\gamma$, TGF- $\beta$, TNF- $\alpha$, IL-1 $\alpha$, IL-1 $\beta$, IL-2, IL-4, IL-6, IL-10, IL-12, IL-1R, IL-1R $\alpha$, IL-4R $\alpha$ ) in patients with MM.

Materials and Methods: EDTA blood was collected from 38 patients with MM included in the study, and DNA was isolated. PCR-SSP method was used for cytokine gene polymorphism typing (Protrans). FISH method was applied using LSI D13S25 SO DNA probe for deletion 13. Results: 13q deletion was detected in 15 of the 38 of the patients. Statistical analysis of $13 \mathrm{q}$ deletion and cytokine gene polymorphisms was not statistically significant $(\mathrm{p}>0.05)$ by patient age, sex, disease stage, and $\beta 2$-microglobulin levels. There is a statistical significance between level of LDH and TGF- $\beta$ TG/TG haplotype $(p=0.002)$. No significant results were obtained in the relationship between cytokine polymorphisms and del13 ( $>0.05)$.

Conclusion: Cytokines, which are associated with the function and viability of plasma cells, have a major role in the etiology and prognosis of the disease. Moreover, similar effects were observed with the $13 \mathrm{q}$ deletion. In our study, we did not find a statistical significance between cytokine gene polymorphisms and $13 \mathrm{q}$ deletion. Although both parameters independently have great importance on the effects of the disease, combining them together does not yield the same effect.

Keywords: Cytokine, polymorphism, multiple myeloma, chromosome 13, deletion

$\ddot{O Z Z}$

Amaç: Multiple myeloma (MM), plazma hücrelerinin kemikiliği, lenfoid dokularveçevrekanında birikmesidir. MM’de görülen en sık anomalilerden biri 13/13q delesyonudur ve yaklaşık \%40-50 oranında görülür. Yapılan çalışmalar, hastaların çoğunda kromozom anomalisinin \%80-90’ının monozomi 13 şeklinde iken \%10-20'sinin bölgesel delesyonlar şeklinde olduğunu göstermiştir. Monozomi 13, sağkalımı belirleyen en önemli belirteçlerden biridir. Sitokinler, immün sistem hücrelerince salınan ve bu hücrelerin birçok fonksiyonunu düzenleyen proteinlerdir. Plazma hücresi, immünglobin üretiminde IL-1 $\beta$, IL-6, IL-10 ve TNF- $\alpha$ gibi sitokinleri üretirler. Tek nükleotid polimorfizmleri de tek amino asit değişikliği ile sitokin fonksiyonunda ve üretiminde farklılıklar oluştururlar. Sitokinler ve kromozom 13 delesyonu (del13), MM hastalığı için önemli olan faktörlerden ikisidir. Biz de çalısmamızda MM ile del13 ve 10 sitokin (IL-1 $\alpha$, IL-1 $\beta$, IL12 , IFN- $\gamma$, TGF- $\beta$, TNF- $\alpha$, IL-2, IL-4, IL-6, IL-10), 2 reseptör (IL-1R, IL-4R $\alpha$ ) ve 1 reseptör antagonistinin (IL-1RA) gen polimorfizmleri arasındaki ilişkiyi incelemeyi amaçladık. 
Gereç ve Yöntem: Çalışmaya multiple myeloma tanısı konmuş 38 hasta dahil edildi. Sitokin gen polimorfizm tiplemesi için PCR-SSP yöntemi kullanıldı (Protrans). Delesyon 13 için LSI D13S25 SO DNA probu kullanılarak FISH yöntemi uygulandı.

Bulgular: Hastaların \%40’ında del 13 saptandı. 13q delesyonu ve sitokin gen polimorfizmlerinin ayrı ayrı hasta yaşı, cinsiyeti, hastalık evresi ve $\beta 2$-mikroglobulin seviyeleri ile istatistiksel analizi sonucu anlamlı bir değer gözlenmedi. LDH düzeyi ile TGF- $\beta$ TG/TG haplotipi arasında istatistiksel anlamlılık saptandı (p:0.002). Sitokin polimorfizmleri ve del13 arasındaki ilişkiye bakıldığında anlamlı bir sonuç elde edilmedi ( $\mathrm{p}>0.05)$.

Sonuç: Sitokin polimorfizmi ve delı3 varlığının hastalığın prognozu üzerine ayrı ayrı etkisi olsa da iki parametrenin birlikte prognoz üzerine etkisi saptanamadı.

Anahtar Kelimeler: Sitokin, polimorfizm, multiple myeloma, kromozom 13, delesyon 


\section{INTRODUCTION}

Multiple myeloma (MM) is a clonal enlargement of plasma cells. This characteristic feature is observed in the bone marrow, lymphoid tissues, and peripheral blood (1). MM is responsible for $15 \%$ of hematological malignancies (2). Factors such as genetic factors, inflammation, and oxidative stress play a role in MM etiology (3).

MM cells are predominantly localized in the bone marrow (BM), and their interaction with bone marrow stromal cells (BMSCs) stimulates the transcription and secretion of cytokines from BMSCs. Although cytokines have a negative effect on MM cells, it is effective as a treatment when applied (4).

Plasma cells increase the release of cytokines such as IL-1 $\beta$, IL-6, and IL-10 (5). IL-6 supports the growth and survival of normal and myeloma plasma cells. IL-10 is a growth factor of malignant plasma cells (4). TGF- $1 \beta$ is an important cytokine in immune regulation (6). Genes of cytokines and their receptors are highly polymorphic. Single nucleotide polymorphisms (SNPs) can affect the expression and/or biological activity of the protein occurring in the gene $(4,7,8)$.

Monosomy 13 is the most common cytogenetic abnormality (approximately $40 \%-50 \%$ ) observed in MM (9-14).

Therefore, cytokines and chromosome 13 deletion (del13) are important prognostic factors for MM. We investigate chromosome 13 deletion and cytokine gene polymorphism in patients with MM.

\section{MATERIALS AND METHODS}

Thirty-eight patients (F/M: 17/21) with MM from İstanbul Faculty of Medicine Hematology were included in the study. Mean age of the patient was 57 years (min:max: 40:78). Analysis of cytokine gene polymorphisms and chromosome 13 abnormality was performed in İstanbul Faculty of Medicine, Department of Medical Biology.

\section{Cytokine gene polymorphism genotyping}

DNA was extracted from the whole peripheral blood with EDTA (15). Cytokine genotyping was performed using the PCR-SSP method (16) with Protrans kit that was designed to detect polymorphisms of thirteen cytokine genes (IL- $1 \alpha$, IL- $1 \beta$, IL-1R, IL1RA, IL2, IL-4, IL-6, IL-10, IL-12, TNF- $\alpha$, INF- $\gamma$, TGF- $\beta$ and IL-4Ra).

\section{Detection chromosome 13 deletion}

Following the FISH procedure, BM or peripheral blood samples were studied. Target DNA was attached to glass slides. The slide containing DNA and probe was denatured in 70\% formamide ( $\mathrm{pH} 7.0$ ) for $5 \mathrm{~min}$ utes at $73^{\circ} \mathrm{C}$. The slides were passed through a cold ethanol batch and allowed to dry. The denatured probe was placed on the target DNA, and the slides were incubated at $37^{\circ} \mathrm{C}$ overnight. After hybridization, it was washed for 2 minutes at $0.5 \mathrm{XSSC}$ at $72^{\circ} \mathrm{C}$ and twice for 3 minutes in phosphate buffered detergent at room temperature. Finally, the slides were stained with DAPI. Thereafter, the slides were viewed with a fluorescence microscope (Nikon E800) with the appropriate set of filters (17).

\section{RESULTS}

Fifteen (40\%) of the 38 patients were detected with chromosome $13 \mathrm{q}$ deletion. No statistical significance between chromosome $13 \mathrm{q}$ deletion and age, gender, stage of disease, level of $\mathrm{LDH}$, and $\beta_{2}$-microglobulin $(p>0.005)$ was found.

There was a statistical significance between level of LDH and TGF- $\beta$ TG/TG haplotype ( $p=0.002$ ). No statistical significance between cytokine gene polymorphisms and age, gender, stage of disease, and level of $\beta_{2}$-microglobulin $(p>0.005)$ was found.

Patients' cytokine gene polymorphisms and chromosome 13q deletion results are shown in Table 1. There was no statistical significance between cytokine gene polymorphisms and $13 q$ deletion $(p>0.05)$.

\section{DISCUSSION}

MM is a malignancy of the plasma cell. The chromosomal abnormalities of MM were detected such as del13 $(40 \%-50 \%)$ and the $t(11 ; 14)(9)$. Deletion of chromosome 13 has been associated with a poor prognosis in MM (18). In this study, we detected 
Table 1. Results of the cytokine gene polymorphisms and $13 \mathrm{q}$ deletion

\begin{tabular}{|c|c|c|c|c|c|c|c|c|c|c|c|c|c|c|}
\hline & $\begin{array}{c}\text { 13q } \\
\text { del }\end{array}$ & $\begin{array}{l}\text { IL- } \\
1 \alpha\end{array}$ & $\begin{array}{l}\text { IL- } \\
1 \beta\end{array}$ & $\begin{array}{l}\text { IL- } \\
1 R\end{array}$ & $\begin{array}{c}\text { IL- } \\
\text { 1RA }\end{array}$ & $\begin{array}{r}\text { IL- } \\
\text { 4RA }\end{array}$ & $\begin{array}{l}\text { IL- } \\
12 \\
\end{array}$ & IFN- $\gamma$ & GF- $\beta 1$ & NF- $\alpha$ & L-2 & IL-4 & L-6 & IL-10 \\
\hline 1 & del- & C-C & $\begin{array}{l}\mathrm{C}-\mathrm{T} / \\
\mathrm{C}-\mathrm{C}\end{array}$ & C & T-C & A-A & A & A-T & G & G & & $\begin{array}{l}\text { TC- } \\
\text { C-TCC }\end{array}$ & & \\
\hline 2 & del- & C-C & $\begin{array}{c}\mathrm{C}-\mathrm{C} / \\
\mathrm{T}-\mathrm{C}\end{array}$ & $-\mathrm{T}$ & T-C & A-A & $1-A$ & $-T$ & G-CG & G-GG & & TC- & AA- & GC- \\
\hline 3 & del- & C-C & $\begin{array}{c}\mathrm{C}-\mathrm{C} / \\
\mathrm{C}-\mathrm{C}\end{array}$ & C-C & T-T & G-A & C-A & -A & G-CG & G-GG & GG- & $\begin{array}{l}\text { GC- } \\
\text { C-TCC }\end{array}$ & $\begin{array}{l}\text { CG- } \\
\text { GG }\end{array}$ & $\begin{array}{c}\text { GC- } \\
\text { C-ACC }\end{array}$ \\
\hline 4 & del- & C-C & $\begin{array}{l}\mathrm{T}-\mathrm{T} / \\
\mathrm{C}-\mathrm{C}\end{array}$ & C-C & T-T & G-A & $-C$ & $-\mathrm{T}$ & G-CC & G-AA & $\begin{array}{c}\text { GG- } \\
\text { TT }\end{array}$ & $\begin{array}{c}\text { TC- } \\
\text { C-TTC }\end{array}$ & $\begin{array}{l}\text { CA- } \\
\text { GG }\end{array}$ & GC- \\
\hline 5 & del- & C-C & $\begin{array}{l}\mathrm{C}-\mathrm{T} / \\
\mathrm{C}-\mathrm{C}\end{array}$ & C-T & T-T & A-A & $1-A$ & $-\mathrm{T}$ & $\mathrm{G}-\mathrm{CC}$ & G-AA & $\begin{array}{c}\text { GG- } \\
\text { TG }\end{array}$ & $\begin{array}{c}\text { GC- } \\
\text { C-TCC }\end{array}$ & $\begin{array}{c}\text { GG- } \\
\text { GG }\end{array}$ & C.110 \\
\hline 6 & del+ & T-C & $\begin{array}{l}\mathrm{C}-\mathrm{T} / \\
\mathrm{C}-\mathrm{C}\end{array}$ & C-C & T-C & A-A & $-\mathrm{A}$ & A-A & $\mathrm{G}-\mathrm{CC}$ & A-GG & GG- & $\begin{array}{c}\text { TC- } \\
\text { C-TTT }\end{array}$ & $\begin{array}{l}\text { GG- } \\
\text { GG }\end{array}$ & $\begin{array}{c}\text { AC- } \\
\text { C-ACC }\end{array}$ \\
\hline 7 & del+ & C-C & $\begin{array}{l}\mathrm{C}-\mathrm{C} / \\
\mathrm{C}-\mathrm{C}\end{array}$ & $-T$ & T-T & A-A & A & A-A & $\mathrm{G}-\mathrm{CC}$ & $\mathrm{G}-\mathrm{AA}$ & & $\begin{array}{c}\text { TC- } \\
\text { C-TTT }\end{array}$ & G- & \\
\hline 8 & del- & T-T & $\begin{array}{c}\mathrm{C}-\mathrm{C} / \\
\mathrm{T}-\mathrm{C}\end{array}$ & C-T & T-C & G-A & $1-A$ & $-T$ & G-CC & G-GG & & $\begin{array}{c}\mathrm{GC} \\
\mathrm{C}-\mathrm{T}\end{array}$ & $\begin{array}{c}\text { GG- } \\
\text { GG }\end{array}$ & \\
\hline 9 & del- & C-C & $\begin{array}{l}\text { T-T / } \\
\text { C-C }\end{array}$ & -C & $\mathrm{T}-\mathrm{C}$ & A-A & -A & $-\mathrm{T}$ & $\mathrm{G}-\mathrm{CC}$ & G-AA & & $\mathrm{T}$ & $\begin{array}{l}\text { CG- } \\
\text { GG }\end{array}$ & \\
\hline 10 & del- & T-C & $\begin{array}{l}\text { C-T / } \\
\text { C-C }\end{array}$ & $-C$ & T-C & $A_{-} A$ & C-A & - T & -CG & A & & & $\begin{array}{l}\text { A- } \\
\text { G }\end{array}$ & \\
\hline 11 & del+ & T-C & $\begin{array}{l}\mathrm{C}-\mathrm{T} / \\
\mathrm{C}-\mathrm{C}\end{array}$ & T-T & T-C & A-A & $-A$ & T-T & -CG & G-GG & & & A- & \\
\hline 12 & del+ & C-C & $\begin{array}{l}\mathrm{C}-\mathrm{T} / \\
\mathrm{C}-\mathrm{C}\end{array}$ & C-T & $\mathrm{T}-\mathrm{T}$ & A-A & $-A$ & $-\mathrm{T}$ & G-CC & G-GG & & $\mathrm{C}$ & A- & \\
\hline 13 & del+ & C-C & $\begin{array}{l}\text { C-C / } \\
\text { C-C }\end{array}$ & C-T & $\mathrm{T}$ & A-A & A & & & & & & $\begin{array}{l}\text { G- } \\
G\end{array}$ & \\
\hline 14 & del- & C-C & $\begin{array}{l}\mathrm{C}-\mathrm{T} / \\
\mathrm{C}-\mathrm{C}\end{array}$ & $\mathrm{T}$ & T-T & G-A & $A$ & $T$ & G & G & & & $\begin{array}{l}\text { CA- } \\
\text { GG }\end{array}$ & \\
\hline 15 & del- & T-C & $\begin{array}{l}\text { C-T / } \\
\text { T-C }\end{array}$ & $-\mathrm{C}$ & T-C & A-A & $-A$ & T & $-\mathrm{CC}$ & GG & G- & $\mathrm{T}$ & $\begin{array}{l}\text { GG- } \\
\text { GG }\end{array}$ & \\
\hline 16 & del+ & C-C & $\begin{array}{l}\text { C-T/ } \\
\text { C-C }\end{array}$ & -C & $\mathrm{T}$ & A-A & -A & & G & G & & & $\begin{array}{l}\text { CA- } \\
\text { GG }\end{array}$ & \\
\hline 17 & d & T-C & $\begin{array}{l}\mathrm{C}-\mathrm{T} / \\
\mathrm{C}-\mathrm{C}\end{array}$ & C-T & T-T & A-A & 1 & & C & A & & & A- & $\begin{array}{c}\text { GC- } \\
\text { C-GCC }\end{array}$ \\
\hline 18 & del+ & C-C & $\begin{array}{l}\mathrm{C}-\mathrm{C} / \\
\mathrm{C}-\mathrm{C}\end{array}$ & $-\mathrm{T}$ & $\mathrm{T}-\mathrm{T}$ & A-A & C-A & & $\mathrm{G}$ & G & & & $\begin{array}{l}\text { GG- } \\
\text { GG }\end{array}$ & \\
\hline 19 & del+ & T-C & $\begin{array}{l}\mathrm{C}-\mathrm{C} / \\
\mathrm{T}-\mathrm{C} \\
\end{array}$ & $-T$ & $\mathrm{~T}-\mathrm{T}$ & G-A & $-A$ & A-T & CG & -GG & & $\mathrm{T}$ & $\begin{array}{l}\text { A- } \\
\text { iG }\end{array}$ & \\
\hline 20 & $\mathrm{de}$ & C-C & $\begin{array}{l}\mathrm{C}-\mathrm{C} / \\
\mathrm{C}-\mathrm{C}\end{array}$ & C-T & $\mathrm{T}$ & $\mathrm{H}$ & -14 & & & G & & & $\begin{array}{l}\text { CA- } \\
\text { GG }\end{array}$ & \\
\hline 21 & de & C-C & & C-C & T-T & A-A & . & & & G & & & $\begin{array}{l}\text { GG- } \\
\text { GG }\end{array}$ & \\
\hline 22 & del- & C-C & $\begin{array}{l}\mathrm{C}-\mathrm{T} / \\
\mathrm{C}-\mathrm{C}\end{array}$ & $-\mathrm{T}$ & T-T & A-A & A-A & A-T & $\mathrm{CC}$ & A & & & $\begin{array}{l}\text { CA- } \\
\text { GG }\end{array}$ & \\
\hline 23 & del- & C-C & $\begin{array}{l}\text { T-T / } \\
\text { C-C }\end{array}$ & C-C & $\mathrm{T}-$ & A-A & -A & & G-CG & G-GG & GG- & TC- & $\begin{array}{l}\text { CA- } \\
\text { CA }\end{array}$ & \\
\hline 24 & del- & T-C & & C & $\mathrm{T}$ & N & r & & CG & G & & & $\begin{array}{l}\text { CA- } \\
\text { GG }\end{array}$ & \\
\hline 25 & de & c & & C-T & $\mathrm{T}-\mathrm{T}$ & G-A & C-C & A-T & TG-CG & G & $\begin{array}{c}\text { GG- } \\
\text { TG }\end{array}$ & & $\begin{array}{l}\text { CA- } \\
\text { GG }\end{array}$ & \\
\hline 26 & del- & T-C & $\begin{array}{l}\mathrm{C}-\mathrm{C} / \\
\mathrm{C}-\mathrm{C}\end{array}$ & C & $\mathrm{T}$ & A-A & H & & $\mathrm{G}-\mathrm{CC}$ & AG-AA & $\begin{array}{l}\text { TG- } \\
\text { TG }\end{array}$ & TC- & $\begin{array}{l}\text { GG- } \\
\text { GG }\end{array}$ & GC- \\
\hline 27 & del+ & T-T & $\begin{array}{l}\text { C-C/ } \\
\text { T-C }\end{array}$ & C-T & T-C & G-A & C-A & A-A & CG-CC & AG-AA & $\begin{array}{l}\text { GG- } \\
\text { TT }\end{array}$ & $\begin{array}{c}\text { GC- } \\
\text { C-TCC }\end{array}$ & $\begin{array}{l}\text { CA- } \\
\text { GG }\end{array}$ & $\begin{array}{c}\text { ATA-A- } \\
\text { TA }\end{array}$ \\
\hline
\end{tabular}


Kekik Çınar Ç, Karahan G, Temurhan S, Kalayoğlu Beşışık S, Savran Oğuz F, Aydın F

Table 1. Continued

\begin{tabular}{|c|c|c|c|c|c|c|c|c|c|c|c|c|c|c|}
\hline 28 & del- & $\mathrm{T}-\mathrm{C}$ & \begin{tabular}{|c|}
$\mathrm{C}-\mathrm{T} /$ \\
$\mathrm{T}-\mathrm{C}$ \\
\end{tabular} & C-C & $\mathrm{T}-\mathrm{C}$ & A-A & A-A & A-T & CG-CG & $\begin{array}{c}\text { GG- } \\
\text { GG }\end{array}$ & $\begin{array}{c}\text { GG- } \\
\text { GG }\end{array}$ & $\begin{array}{c}\text { TC- } \\
\text { C-TCC }\end{array}$ & $\begin{array}{l}\text { CA- } \\
\text { GG } \\
\end{array}$ & $\begin{array}{c}\text { GC- } \\
\text { C-GCC }\end{array}$ \\
\hline 29 & del- & $\mathrm{T}-\mathrm{C}$ & $\begin{array}{l}\mathrm{C}-\mathrm{T} / \\
\mathrm{T}-\mathrm{C}\end{array}$ & $\mathrm{C}-\mathrm{T}$ & $\mathrm{T}-\mathrm{T}$ & G-A & C-A & $\mathrm{T}-\mathrm{T}$ & TG-CG & $\begin{array}{l}\text { GG- } \\
\text { GG }\end{array}$ & $\begin{array}{l}\text { GG- } \\
\text { TG }\end{array}$ & $\begin{array}{c}\text { TC- } \\
\text { C-TCC }\end{array}$ & $\begin{array}{l}\text { CA- } \\
\text { GG }\end{array}$ & $\begin{array}{l}\text { ATA- } \\
\text { GCC }\end{array}$ \\
\hline 30 & del- & C-C & $\begin{array}{l}\mathrm{T}-\mathrm{T} / \\
\mathrm{C}-\mathrm{C}\end{array}$ & $\mathrm{C}-\mathrm{T}$ & $\mathrm{T}-\mathrm{T}$ & A-A & C-A & $\mathrm{T}-\mathrm{T}$ & TG-CG & $\begin{array}{l}\text { GG- } \\
\text { GG }\end{array}$ & $\begin{array}{l}\text { TG- } \\
\text { TG }\end{array}$ & $\begin{array}{c}\text { TC- } \\
\text { C-TCC }\end{array}$ & $\begin{array}{l}\text { GG- } \\
\text { GG }\end{array}$ & $\begin{array}{c}\text { GC- } \\
\text { C-ACC }\end{array}$ \\
\hline 31 & del+ & $\mathrm{T}-\mathrm{C}$ & $\begin{array}{l}\mathrm{C}-\mathrm{T} / \\
\mathrm{C}-\mathrm{C}\end{array}$ & $\mathrm{C}-\mathrm{C}$ & $\mathrm{T}-\mathrm{C}$ & A-A & A-A & A-T & TG-CG & $\begin{array}{c}\text { AG- } \\
\text { GG }\end{array}$ & $\begin{array}{l}\text { TG- } \\
\text { TG }\end{array}$ & $\begin{array}{c}\text { GC- } \\
\text { C-GCC }\end{array}$ & $\begin{array}{l}\text { CA- } \\
\text { CA }\end{array}$ & $\begin{array}{c}\text { GC- } \\
\text { C-GCC }\end{array}$ \\
\hline 32 & del- & C-C & $\begin{array}{l}\mathrm{T}-\mathrm{T} / \\
\mathrm{C}-\mathrm{C}\end{array}$ & C-C & $\mathrm{T}-\mathrm{T}$ & A-A & A-A & A-A & CG-CG & $\begin{array}{l}\text { GA- } \\
\text { GG }\end{array}$ & $\begin{array}{l}\text { GG- } \\
\text { TG }\end{array}$ & $\begin{array}{c}\text { GC- } \\
\text { C-TTT }\end{array}$ & $\begin{array}{l}\text { CG- } \\
\text { GG }\end{array}$ & $\begin{array}{l}\text { ATA- } \\
\text { ACC }\end{array}$ \\
\hline 33 & del+ & $\mathrm{C}-\mathrm{C}$ & $\begin{array}{l}\mathrm{C}-\mathrm{T} / \\
\mathrm{C}-\mathrm{C}\end{array}$ & C-C & $\mathrm{T}-\mathrm{T}$ & G-A & C-A & $\mathrm{T}-\mathrm{T}$ & CG-CC & $\begin{array}{l}\text { GG- } \\
\text { GG }\end{array}$ & $\begin{array}{l}\text { GG- } \\
\text { TT }\end{array}$ & $\begin{array}{c}\text { TC- } \\
\text { C-GCT }\end{array}$ & $\begin{array}{l}\text { CG- } \\
\text { GG }\end{array}$ & $\begin{array}{l}\text { ATA- } \\
\text { GCC }\end{array}$ \\
\hline 34 & del- & C-C & $\begin{array}{l}\mathrm{T}-\mathrm{T} / \\
\mathrm{C}-\mathrm{C}\end{array}$ & C-C & $\mathrm{C}-\mathrm{C}$ & A-A & A-A & A-A & CG-CC & $\begin{array}{l}\text { AG- } \\
\text { AA }\end{array}$ & $\begin{array}{l}\text { GG- } \\
\text { TG }\end{array}$ & $\begin{array}{c}\text { GC- } \\
\text { C-TCC }\end{array}$ & $\begin{array}{l}\text { GG- } \\
\text { GG }\end{array}$ & $\begin{array}{l}\text { ATA- } \\
\text { ACC }\end{array}$ \\
\hline 35 & del- & $\mathrm{T}-\mathrm{C}$ & $\begin{array}{l}\mathrm{C}-\mathrm{T} / \\
\mathrm{C}-\mathrm{C}\end{array}$ & $\mathrm{T}-\mathrm{T}$ & $\mathrm{T}-\mathrm{C}$ & A-A & A-A & $A-T$ & TG-CG & $\begin{array}{l}\text { GG- } \\
\text { GG }\end{array}$ & $\begin{array}{l}\text { GG- } \\
\text { GG }\end{array}$ & $\begin{array}{c}\text { GC- } \\
\text { C-TCC }\end{array}$ & $\begin{array}{l}\text { CG- } \\
\text { GG }\end{array}$ & $\begin{array}{c}\text { GC- } \\
\text { C-ACC }\end{array}$ \\
\hline 36 & del+ & C-C & \begin{tabular}{l|}
$\mathrm{C}-\mathrm{C} /$ \\
$\mathrm{C}-\mathrm{C}$
\end{tabular} & $\mathrm{T}-\mathrm{T}$ & $\mathrm{C}-\mathrm{C}$ & A-A & C-A & $\mathrm{T}-\mathrm{T}$ & CG-CC & $\begin{array}{c}\text { AG- } \\
\text { AA }\end{array}$ & $\begin{array}{l}\text { GG- } \\
\text { GG }\end{array}$ & $\begin{array}{c}\text { GC- } \\
\text { C-TCC }\end{array}$ & $\begin{array}{l}\text { CA- } \\
\text { GG }\end{array}$ & $\begin{array}{c}\text { GC- } \\
\text { C-ACC }\end{array}$ \\
\hline 37 & del- & $\mathrm{T}-\mathrm{C}$ & \begin{tabular}{|c|}
$\mathrm{C}-\mathrm{T} /$ \\
$\mathrm{T}-\mathrm{C}$
\end{tabular} & C-C & $\mathrm{C}-\mathrm{C}$ & G-A & A-A & $A-T$ & TG-TG & $\begin{array}{l}\text { GG- } \\
\text { GG }\end{array}$ & $\begin{array}{l}\text { GG- } \\
\text { TG }\end{array}$ & $\begin{array}{c}\text { TC- } \\
\text { C-TTT }\end{array}$ & $\begin{array}{l}\text { CA- } \\
\text { GG }\end{array}$ & $\begin{array}{c}\text { GC- } \\
\text { C-ACC }\end{array}$ \\
\hline 38 & del+ & $\mathrm{T}-\mathrm{C}$ & $\begin{array}{l}\mathrm{C}-\mathrm{T} / \\
\mathrm{C}-\mathrm{C}\end{array}$ & $\mathrm{C}-\mathrm{T}$ & $\mathrm{T}-\mathrm{C}$ & G-A & A-A & A-A & CG-CC & $\begin{array}{l}\text { AG- } \\
\text { AA }\end{array}$ & $\begin{array}{l}\text { TG- } \\
\text { TT }\end{array}$ & $\begin{array}{c}\text { GC- } \\
\text { C-TCC }\end{array}$ & $\begin{array}{l}\text { CG- } \\
\text { GG }\end{array}$ & $\begin{array}{c}\text { GC- } \\
\text { C-GCC }\end{array}$ \\
\hline
\end{tabular}

chromosome 13 deletion rate at $40 \%$, which did not demonstrate statistical significance between chromosome 13q deletion and age, gender, stage of disease, level of $\mathrm{LDH}$, and $\beta_{2}$-microglobulin.

Cytokines are crucial in the regulation of key pathways of immunity. SNPs alter the expression or function of the gene product. Some studies have shown an association between polymorphisms in cytokine genes and MM $(4,5,19)$; however, other studies have not detected it $(6,20,21)$. No statistical significance was found between cytokine gene polymorphisms and age, gender, stage of disease, and level of $\beta_{2}$-microglobulin. However, a statistical significance was observed between high levels of $\mathrm{LDH}$, which is the indicator of a poor prognosis and TGF- $\beta$ TG/TG haplotype. Furthermore, TG/TG haplotype associates with high level production of TGF- $\beta$. We considered that TGF- $\beta$ triggers conversion to plasma cells from $B$ cells by elevating LDH levels, and therefore, cause to a poor prognosis for the disease.

Cytokines, which is related the function and survive of plasma cells and $13 \mathrm{q}$ deletion, have a major role in the etiology and prognosis of diseases. In our study, a statistical significance between cytokine gene polymorphisms and $13 \mathrm{q}$ deletion was observed. $\mathrm{Al}-$ though both parameters independently have great importance on the effects of the disease, it seems that combining them together is not favorable.

Peer Review: Externally peer-reviewed.

Hakem Değerlendirmesi: Dış bağımsız.

Author Contributions: Conception/Design of Study- Ç.K.Ç., G.K., S.T.; Data Acquisition- Ç.K.Ç., G.K., S.T; Data Analysis/Interpretation- Ç.K.Ç., G.K., S.T., S.K.B., F.S.O., F.A.; Drafting Manuscript- Ç.K.Ç., G.K., S.T.; Critical Revision of Manuscript- Ç.K.Ç., S.K.B., F.S.O.,F.A.; Final Approval and Accountability- Ç.K.Ç., G.K., S.T., S.K.B., F.S.O., F.A.; Technical or Material Support- Ç.K.Ç., G.K., S.T.; SupervisionÇ.K.Ç., S.K.B., F.S.O., F.A.

Yazar Katkıları: Çalışma Konsepti/TasarımÇ.K.Ç., G.K., S.T.; Veri Toplama- Ç.K.Ç., G.K., S.T ; Veri Analizi/Yorumlama- Ç.K.Ç., G.K., S.T., S.K.B., F.S.O., F.A.; Yazı Taslağı- Ç.K.Ç., G.K., S.T.; İçeriğin Eleştirel İncelemesi- Ç.K.Ç., S.K.B., F.S.O.,F.A.; Son Onay ve Sorumluluk- Ç.K.Ç., G.K., S.T., S.K.B., F.S.O., F.A.; Malzeme ve Teknik Destek- Ç.K.Ç., G.K., S.T.; Süpervizyon- Ç.K.Ç., S.K.B., F.S.O., F.A.

Conflict of Interest: Authors declared no conflict of interest. 
Çıkar Çatışması: Yazarlar çıkar çatışması beyan etmemişlerdir

Financial Disclosure: Authors declared no financial support.

Finansal Destek: Yazarlar finansal destek beyan etmemişlerdir.

\section{REFERENCES}

1. Caligaris CF, Gregoretti MG, Merico F, Gottardi D, Ghia P, Parvis G, et al. Bone marrow microenvironment and the progression of multiple myeloma. Leuk Lymphoma 1992;8(12):15-22.

2. Angtuago EJ, Fassas AB, Walker R, Sethi R, Barlogie B. Multiple myeloma: clinical review and diagnostic imaging. Radiology 2004;231(1):1123.

3. Stellrecht CM, Gandhi V. Myeloma antioxidant status: the good, the bad and reactive. Leuk Lymphoma 2009;50(5):691-3.

4. Banu C, Moise A, Arion CR, Coriu D, Tanase A, Constantinescu I. Cytokine gene polymorphisms support diagnostic monitoring of Romanian multiple myeloma patients. J Med Life 2011;4(3):264-8.

5. Vangsted AJ, Klausen TW, Ruminski W, Gimsing P, Andersen NF, Gang AO, et al. The polymorphism IL-1 beta T-31C is associated with a longer overall survival in patients with multiple myeloma undergoing auto-SCT. Bone Marrow Transp 2009;43(7):539-45.

6. Wang H, Gao C, Xu L, Yang Z, Zhao W, Kong X. Laboratory characterizations on 2007 cases of monoclonal gammopathies in east china. Cell Mol Immunol 2008;5(4):293-8.

7. Leigh JK. The extent and analysis of cytokine and cytokine receptor gene polymorphism. Transplan Immunol 2002;10:143-6.

8. Ollier WE. Cytokine genes and disease susceptibility. Cytokine 2004;28(4-5):174-8.

9. Ho PJ. Chromosomal and genetic abnormalities in myeloma. Clin Lab Haem 2002;24(5):259-69

10. Liebisch P, Wendl C, Wellmann A, Kröber A, Schilling G, Goldschmidt $\mathrm{H}$, et al. High incidence of trisomies 1q, 9q, and 11q in multiple myeloma: results from a comprehensive molecular cytogenetic analysis. Leukemia 2003;17:2535-7.

11. Perez-Simon JA, Garcia-Sanz R, Tabernero MD, Bladé J, Moro MJ, Fernández-Calvo J, et al. Prognostic value of numerical chromosome aberrations in multiple myeloma: A FISH analysis of 15 different chromosomes. Blood 1998;91(9):3366-71.

12. Zojer N, Königsberg R, Ackermann J, Fritz E, Dallinger S, Krömer E, et al. Deletion of 13q14 remains an independent adverse prognostic variable in multiple myeloma despite its frequent detection by interphase fluorescence in situ hybridization. Blood 2000;95(6):1925-30.

13. Facon T, Avet-Loiseau H, Guillerm G, Moreau $\mathrm{P}$, Geneviève F, Zandecki M, et al. Chromosome 13 abnormalities identified by FISH analysis and serum B-2-microglobulin produce a powerful myeloma staging system for patients receiving high-dose therapy. Blood 2001;97(6):1566-71.

14. Fonseca R, Harrington D, Oken M, Dewald GW, Bailey RJ, Van Wier SA, et al. Biologic and prognostic significance of interphase FISH detection of chromosome 13 abnormalities (D13) in multiple myeloma: an Eastern Cooperative Oncology Group (ECOG) Study. Cancer Res 2002;62(3):715-20.

15. Gustincich S, Manfiolett G, Del Sal G, Schneider C, Carninci P. A fast method for high-quality genomic DNA extraction from whole human blood. Bio Techniques 1991;11(3):298-302.

16. Olerup O, Zetterquist H. DR low resolution PCR-SSP typing - a correction and an up-date. Tissue Antigens 1993;41(1):55-6.

17. Özdilli K, Aydın F, Beşışık SK, Oğuz F, Temurhan $S$, Çako Ö, et al. Detection of chromosomal abnormalities on interface cells from myeloid leukemias by FISH. Nobel Med 2010; 6(3): 84-89.

18. Fonseca R, Oken MM, Harrington D, Bailey RJ, Van Wier SA, Henderson KJ, et al. Deletions of chromosome 13 in multiple myeloma identified by interphase FISH usually denote large deletions of the q arm or monosomy. Leukemiz 2001;15(6):981-6. 
19. Chakraborty B, Vishnoi G, Gowda SH, Goswami B. Interleukin-6 gene-174 G/C promoter polymorphism and its association with clinical profile of patients with multiple myeloma. AsiaPac J Clin Oncol 2014;13(5):e402-e407.

20. G Mazur, K Bogunia-Kubik, T Wróbel, L Karabon, M Polak, K Kuliczkowski, et al. IL-6 and IL-10 promoter gene polymorphisms do not associate with the susceptibility for multiple myeloma. Immunol Lett 2005;96(2):241-6.

21. Iakupova EV, Grinchuk OV, Kalimullina D, Bakirov B, Galimova RR, Makarova $\mathrm{OV}$, et al. Molecular genetic analysis of the Interleukin 6 and Tumor Necrosis Factor a gene polymorphisms in multiple myeloma. Mol Biol (Mosk) 2003;37:420-4. 